\title{
EDITORIAL
}

\section{Towards the development of EU/EEA Standards for Tuberculosis Care (ESTC)}

\author{
G.B. Migliori*, G. Sotgiü ${ }^{\#}$, F. Blasi ${ }^{\ddagger}$, A. Zumla ${ }^{+}$, R. Loddenkemper ${ }^{\S}$, M.C. Raviglione $^{f}$, I. Abubakar**, \\ A. Sandgren $\# \#$ and D. Manissero \#\#
}

$\mathbf{T}$ uberculosis (TB) continues to pose a public-health threat to the people of the member states of the European Union (EU) and European Economic Area (EEA). EU/EEA countries comprise Austria, Belgium, Bulgaria, Cyprus, the Czech Republic, Denmark, Estonia, Finland, France, Germany, Greece, Hungary, Iceland, the Republic of Ireland, Italy, Latvia, Liechtenstein, Lithuania, Luxembourg, Malta, the Netherlands, Norway, Poland, Portugal, Romania, Slovakia, Slovenia, Spain, Sweden and the UK. In 2009, almost 80,000 TB cases were notified in the EU/EEA, and while several countries are progressing towards achieving and sustaining low levels of TB incidence, the contrasts in TB disease burden remain great within the EU/EEA [1]. Some countries are faced with a significant burden of drugresistant TB, and multidrug-resistant (MDR)-TB. In 2009, 5.3\% of all active TB cases reported in the EU/EEA were MDR-TB, and $14.1 \%$ were resistant to any of the first-line TB drugs [1]. HIV coinfection is still low, with an overall proportion of $2.3 \%$ of $\mathrm{TB}$ cases being HIV-seropositive, although accurate surveillance data reporting is suboptimal in many countries [1-3].

\section{TB CONTROL AND ELIMINATION STRATEGIES IN THE EU/EEA}

The EU/EEA member states were proactive in adopting the existing global strategies for TB control, including Directly Observed Treatment Short Course (DOTS) and the Stop TB Strategy [4]. The stepwise adoption of these principles was facilitated by the development of a set of European-specific, consensus-based documents born within the Wolfheze initiative [5]. A European group of experts, country representatives and international organisations (World Health Organization (WHO), International Union Against Tuberculosis and Lung Disease (IUATLD) and the KNCV Tuberculosis Foundation) developed guidance and policy documents on TB control and elimination, surveillance, screening and management of

\footnotetext{
*WHO Collaborating Centre for TB and Lung Diseases, Fondazione S. Maugeri, Care and Research Institute, Tradate, ${ }^{*}$ Hygiene and Preventive Medicine Institute, University of Sassari, Sassari, and "Respiratory Medicine Section, Dipartimento Toraco-Polmonare e Cardiocircolatorio, University of Milan, IRCCS Fondazione Cà Granda, Milan, Italy. ${ }^{+}$Division of Infection and Immunity, Dept of Infection, University College London Medical School, London, and **Tuberculosis Section, Health Protection Agency, Colindale, UK. ${ }^{s}$ German Central Committee against Tuberculosis (DZK), Berlin Germany. ${ }^{f}$ World Health Organization, Geneva, Switzerland. ${ }^{\# \#}$ European Centre for Disease Prevention and Control (ECDC), Stockholm, Sweden.
}

CORRESPONDENCE: G.B. Migliori, WHO Collaborating Centre for TB and Lung Diseases, Fondazione S. Maugeri, Via Roncaccio 16, Tradate, Varese, 21049, Italy. E-mail: giovannibattista. migliori@fsm.it immigrants, treatment outcome monitoring, drug susceptibility testing [6-10] and, more recently, contact tracing [11]. The whole package was then combined into a single, comprehensive European framework for TB control and elimination in low ТВ burden countries, paying particular attention to peculiar issues such as TB among migrants and vulnerable groups [12]. In 2008, the European Centre for Disease Prevention and Control (ECDC) developed the Framework Action Plan to Fight TB in the EU and more recently an EU/ EEA relevant monitoring framework [13, 14].

\section{FROM GUIDELINES TO STANDARDS}

The International Standards for Tuberculosis Care (ISTC) [15, 16] were first developed in 2006 by 27 experts from 14 countries [15]. These were revised in 2009 by 50 experts from 15 countries and included the perspectives of several organisations and international societies that endorsed them for universal use [16].

The ISTC prescribe a widely accepted level of TB care which guides all healthcare providers and clinicians, both public and private, in achieving optimal standards in managing patients who have, or are suspected of having, active TB. The 21 standards of the ISTC's second version [16], are organised into four sections: 1) standards for diagnosis; 2) standards for treatment; 3) standards for addressing HIV infection and other comorbid conditions; and 4) standards for public health. These are still valid and applicable in the EU/EEA. The ISTC complement existing national or international guidelines, and are consistent with $\mathrm{WHO}$ definitions and recommendations. The first edition of the ISTC stated that: "The Standards should be viewed as a living document that will be revised as technology, resources and circumstances change" [15].

Despite efforts to introduce ISTC among EU/EEA healthcare providers (including clinicians), their widespread usage has been suboptimal. A more tailored set of standards that could potentially improve acceptability and utility among clinical networks in the EU/EEA is required. Several other justifications for the development of new standards specific for the countries of the EU/EEA are given in table 1. It is against this background that a process, led jointly by the ECDC and the European Respiratory Society (ERS), has been initiated to adapt the ISTC to the EU/EEA setting. This has led to the drafting of EU/EEA Standards for Tuberculosis Care (ESTC). Realising, within this initiative, the different scope and nature of the two organisations, each has taken responsibility for its respective mandate and expertise. Specifically, ERS has taken 


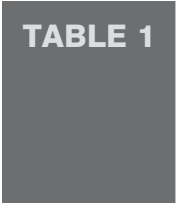

\section{Justification for the development of European Union (EU)/European Economic Area (EEA)- specific standards in the area of diagnosis, treatment, HIV/tuberculosis (TB) and comorbidities, public health and prevention}

The majority of EU/EEA countries have a low incidence of TB. However, a heterogeneous setting exists: some countries have high and intermediate levels of TB, with varying levels of MDR-TB and HIV/TB co-infection; and some countries border non-EU countries with a higher TB and MDR-TB burden.

TB services are fully integrated and merged within the health system in the majority of EU/EEA countries. This presents peculiarities in allocating responsibilities for the delivery of TB care.

The EU/EEA countries have a long-established tradition of TB control that has evolved over past decades. Implementation of new tools and a high standard of diagnosis and care is often present in EU countries. The EU/EEA countries are committed to pursue elimination, sharing a common platform (based on the Wolfheze documents and the EU Framework action plan) and the ECDC-driven surveillance system. In spite of efforts to introduce ISTC among EU/EEA healthcare providers (including clinicians), their implementation has been suboptimal. A more tailored set of standards could potentially improve acceptability among clinical networks.

MDR: multidrug-resistant; ECDC: European Centre for Disease Prevention and Control; ISTC: International Standards for Tuberculosis Care.

the lead in developing, and will assure endorsement of, the clinically related standards. ECDC has led the development of the public-health standards and will ensure endorsement of these through its specific competent channels, such as its advisory forum.

The draft ESTC have been specifically developed and drafted to complement the ISTC and other existing guidelines. The draft EU/EEA standards are based mainly, but not exclusively, on existing gaps in case management as identified through a recent European MDR-TB case management survey [17-20] and the survey of TB surveillance systems in low-incidence countries, including countries of the EU/EEA [21].

\section{TOWARDS THE DEVELOPMENT OF ESTC}

The ESTC draft document comprises standards related to the four clinical activities as outlined in table 2 (those related to diagnosis, treatment, and HIV/TB and comorbidity clinical management were developed by the ERS, while ECDC drafted standards related to public health). The draft document benefited from the collaboration of a panel of 30 experts, including ERS experts (Chair of the ERS Scientific Committee, Head of the Respiratory Infections Assembly, Chair of Study Group 10.2 Tuberculosis, ERS Vice-President, ERS Guidelines Coordinator and a Chief Editor of the European Respiratory Journal (ERJ)), ECDC experts, national TB programmes (Belgium, Denmark, Estonia, Germany, Italy, Latvia, the Netherlands, Portugal, Romania, Spain, Switzerland, the UK and the USA), international societies (American Thoracic Society), civil society representatives (affected communities) and international organisations (WHO, the IUATLD Europe Region and KNCV Tuberculosis Foundation).

The process was specifically designed to ensure acceptability of the evidence-based document by over 10,000 ERS members globally and by the ERS representatives, officers and staff, and a wide audience represented by national experts, members of affected communities and international organisations, thus increasing the potential impact of the document once finalised and published. The 2006 and 2009 ISTC documents [15, 16] were used as the basis to develop the draft ESTC, as were the ECDC survey on TB/MDR-TB in the EU/EEA [17-20], the ERS TB management guidelines [22], and the full series of Wolfheze policy documents [5-12]. In addition, a nonsystematic review of the evidence relevant for the development of ESTC was performed (including the peer-reviewed manuscripts and systematic reviews, and meta-analyses included in the ISTC document and those published after the second edition of the ISTC). This reviewed all manuscripts identifying pitfalls in TB management in the EU/EEA setting.

A Delphi process was performed to define the list of standards based on the priority areas identified by the previously mentioned ECDC-funded case management survey [17-20]. These areas covered both clinical management and publichealth perspectives. The panel of experts were asked to assign priorities to the first draft of 45 proposed standards and to

TABLE 2 Rationale for the four key components of the European Union (EU)/European Economic Area (EEA) Standards for Tuberculosis Care (ESTC)

Diagnosis

Treatment

HIV/TB and comorbidities

Public health and prevention
The ESTC are based not only on TB suspects identified through sputum smear microscopy, but are adapted to the EU/EEA setting including culture, DST and rapid testing including rapid identification for rifampicin resistance. This is consistent with the culture-based definition used in the EU/EEA.

Given the availability of rapid diagnostic tests for TB and rifampicin resistance, the need to start MDR treatment from the beginning, under high-quality management conditions.

To ensure quality treatment monitoring based on culture.

Universal access to anti-retroviral treatment is available to all HIV infected people, as is the capacity to detect all cases. This impacts on the management of provision of IPT.

Not only HIV-infected individuals, but also those with a range of immunosuppressive conditions (such as TNF- $\alpha$ antagonist, corticosteroid, cancer treatment, diabetes, intravenous drug use and alcohol abuse) should be evaluated for IPT and proper follow-up for TB.

In the absence of vertical TB programmes and with the full integration of services, healthcare providers are assuming an important responsibility for ensuring key public health and infection control functions related to detection and prevention of TB

TB: tuberculosis; DST: drug susceptibility testing; MDR: multidrug resistant; IPT: isoniazid preventive therapy; TNF: tumour necrosis factor. 
complete the literature synopsis of the standards-supporting evidence with a score ranging from 5 (high relevance) to 1 (low relevance). Based on the results of the Delphi process, the draft concept standards and the literature synopsis were completed and circulated within the panel of experts for two rounds of review, comments and additions. Consensus was reached to formulate the standards around four sections (diagnosis, treatment, clinical management of TB/HIV and comorbidities, and public health and prevention) encompassing all the 45 previously identified standards. The Delphi exercise was completed by $85 \%$ of the panel experts who agreed to participate, with the main score per area being high, ranging 4.04-4.74. The literature synopsis was also scored and 21 references were added to the 97 initially proposed. The ESTC target all healthcare providers and particularly clinicians. The standards are complemented by supporting enablers, making the document as comprehensive, targeted and clear as possible.

The concept of ESTC was first presented and discussed in a symposium at the 2010 ERS Congress in Barcelona. Three working meetings involving ERS and the ECDC experts and a vis-à-vis meeting of the writing committee were organised in 2011, preceding circulation of the draft document to the expert panel. The draft ESTC document will be presented and discussed further during a symposium of the 2011 ERS Congress, to be held in Amsterdam in September 2011. The pre-final version of the ESTC will be completed in October 2011, before going through an official endorsement process of the ERS and the ECDC Advisory Forum for the clinical and public health standards, respectively. The final ESTC document is scheduled to be launched at the World TB Day 2012 as a peer-reviewed article to be published in the ERJ. The responsibility of each of the two organisations involved will remain clearly identifiable in the presentation of the clinical and public health standards.

It is envisaged that the ESTC will be widely distributed to all healthcare providers and implemented throughout the EU/EEA.

\section{CONCLUSION}

The EU/EEA is faced with an emerging problem of drugresistant TB. Thus, the highest possible standard of care needs to be provided to TB patients diagnosed each year in EU/EEA countries. The EU/EEA standards are being designed specifically to complement the ISTC. The ESTC will offer healthcare providers standards of care tailored to the needs of the EU/ EEA, guiding them to provide the best possible care for all TB suspects and patients.

\section{STATEMENT OF INTEREST}

None declared.

\section{REFERENCES}

1 European Centre for Disease Prevention and Control/World Health Organization Regional Office for Europe. Tuberculosis Surveillance in Europe 2009. Stockholm, European Centre for Disease Prevention and Control, 2011.

2 Pimpin L, Drumright LN, Kruijshaar ME, et al. TB HIV co-infection in EU/EEA states. Eur Respir J 2011; [Epub ahead of print DOI: 10.1183/09031936.00198410].

3 Kruijshaar ME, Pimpin L, Abubakar I, et al. The burden of TB-HIV in the European Union - how much do we know? A survey of
TB-HIV surveillance practices and results. Eur Respir J 2011; [Epub ahead of print. DOI: 10.1183/09031936.00198310].

4 Raviglione MC, Uplekar MW. WHO's new Stop TB Strategy. Lancet 2006; 367: 952-954.

5 Veen J, Migliori GB, Raviglione M, et al. Harmonisation of TB control in the WHO European region: the history of the Wolfheze Workshops. Eur Respir J 2011; 37: 950-959.

6 Clancy L, Rieder HL, Enarson DA, et al. Tuberculosis elimination in the countries of Europe and other industrialized countries. Eur Respir J 1991; 4: 1288-1295.

7 Rieder HL, Watson JM, Raviglione MC, et al. Surveillance of tuberculosis in Europe. Eur Respir J 1996; 9: 1097-1104.

8 Rieder HL, Zellweger J-P, Raviglione MC, et al. Tuberculosis control in Europe and international migration. Eur Respir J 1994; 7: 1545-1553.

9 Veen J, Raviglione M, Rieder HL, et al. Standardized tuberculosis treatment outcome monitoring in Europe. Recommendations of a Working Group of the World Health Organization (WHO) and the European Region of the International Union Against Tuberculosis and Lung Disease (IUATLD) for uniform reporting by cohort analysis of treatment outcome in tuberculosis patients. Eur Respir $J$ 1998; 12: 505-510.

10 Schwoebel V, Lambregts-van Weezenbeek CS, Moro ML, et al. Standardization of antituberculosis drug resistance surveillance in Europe. Recommendations of a World Health Organization (WHO) and International Union Against Tuberculosis and Lung Disease (IUATLD) Working Group. Eur Respir J 2000; 16: 364-371.

11 Erkens CG, Kamphorst M, Abubakar I, et al. Tuberculosis contact investigation in low prevalence countries: a European consensus. Eur Respir J 2010; 36: 925-949.

12 Broekmans JF, Migliori GB, Rieder HL, et al. European framework for tuberculosis control and elimination in counties with a low incidence. Recommendations of the World Health Organization (WHO), International Union Against Tuberculosis and Lung Disease (IUATLD) and Royal Netherlands Tuberculosis Association (KNCV) Working Group. Eur Respir J 2002; 19: 765-775.

13 European Centre for Disease Prevention and Control. Framework Action Plan to Fight Tuberculosis in the European Union. Stockholm, European Centre for Disease Prevention and Control, 2008.

14 European Centre for Disease Prevention and Control. Progressing Towards TB Elimination. Stockholm, European Centre for Disease Prevention and Control, 2008.

15 Tuberculosis Coalition for Technical Assistance. International Standards for Tuberculosis Care (ISTC). The Hague, Tuberculosis Coalition for Technical Assistance, 2006

16 Tuberculosis Coalition for Technical Assistance. International Standards for Tuberculosis Care (ISTC). 2nd Edn. The Hague, Tuberculosis Coalition for Technical Assistance, 2009.

17 Sotgiu G, Centis R, D'Ambrosio L, et al. Development of a standardised tool to survey MDR-/XDR-TB case management in Europe. Eur Respir J 2010; 36: 208-211.

18 Sotgiu G, D'Ambrosio L, Centis R, et al. TB and M/XDR-TB infection control in European TB reference centres: the Achilles's heel? Eur Respir J 2011; (in press).

19 Sotgiu G, Centis R, D'Ambrosio L, et al. Development of a standardized tool to survey MDR-/XDR-TB case management in Europe. Eur Respir J 2010; 36: Suppl. 54, 32s.

20 Facchini AL, D'Ambrosio L, Sotgiu G, et al. Is management of MDR-/XDR-TB in Europe adequate? Eur Respir J 2010; 36: Suppl. $54,32 \mathrm{~s}$.

21 Mor Z, Migliori GB, Althomsons SP, et al. Comparison of tuberculosis surveillance systems in low-incidence industrialised countries. Eur Respir J 2008; 32: 1616-1624.

22 Migliori GB, Raviglione MC, Schaberg T, et al. Tuberculosis management in Europe. Task Force of the European Respiratory Society (ERS), the World Health Organization (WHO) and the International Union against Tuberculosis and Lung Disease (IUATLD) Europe Region. Eur Respir J 1999; 14: 978-992. 